\title{
Probing the Structure and Kinematics of Outflows in Episodic Accretion of YSOs
}

\author{
J. P. Ninan* \\ Tata Institute of Fundamental Research, Homi Bhabha Road, Colaba, Mumbai-400005, India \\ E-mail: ninanetifr.res.in \\ D. K. Ojha \\ Tata Institute of Fundamental Research, Homi Bhabha Road, Colaba, Mumbai-400005, India \\ E-mail: ojha@tifr.res.in
}

\section{Mohan}

Inter-University Centre for Astronomy and Astrophysics, Ganeshkhind, Pune-411007, India

E-mail: vmohan@iucaa.ernet.in

\begin{abstract}
The driving mechanism of the outflows in young low-mass stars is an open question in star formation. The innermost part near the central proto-star, where the outflows originate, can be studied using high resolution spectroscopy. We present here our results from SALT-HRS instrument to resolve the structures in the outflows from a young stellar object undergoing an accretion outburst (V899 Mon). Our high resolution (R 37000) optical spectrum could resolve multi-velocity component structures in the outflow traced by blue-shifted absorption in $\mathrm{H} \alpha$ and Ca II IR triplet lines. They are more consistent with magnetic reconnection winds than steady stellar outflows. We could also resolve the forbidden lines from the shocked regions in jets/outflows revealing a peculiar plateau structure.
\end{abstract}

SALT Science Conference 2015 -SSC2015-

1-5 June, 2015

Stellenbosch Institute of Advanced Study, South Africa

\footnotetext{
* Speaker.
} 


\section{Introduction}

Accretion in young low-mass stars from their protoplanetary discs has recently been found to be episodic in nature by various studies. These low-mass sources which are undergoing short duration (years/decades) of heavy accretion (a factor of 10 to 100 increase in accretion rate) are known as FUors/EXors. These episodic outbursts could potentially solve some of the open issues in star formation like the "Luminosity Problem" [4], the origin of knots in the outflows/jets from young stellar objects (YSOs) [5], silicate crystallization in protoplanetary discs [1] etc. Episodic accretion can also significantly change the pre-main sequence isochrones used extensively for initial mass function and age/mass estimation [2]. From their number statistics it is estimated that every low-mass star will undergo $\sim 50$ such short duration outbursts during its formation stage [8]. But the short duration of outburst (months/years) with respect to million year timescale of star formation, makes these outbursts extremely rare to detect in star-forming regions. The collimated jet launching mechanism in YSOs is still not fully understood. It has been proposed by various models that the outflows are correlated with the accretion. The protoplanetary discs of FUors/EXors are the perfect site to test these models as we can monitor the change in accretion rate and its effect on outflows from spectroscopic observations of the same source in short timescales.

With SALT's newly installed HRS spectrograph, we could resolve the structure and kinematics of the outflows originating from the innermost magneto-spheric region of accretion. We present here the resolved ( $\mathrm{R}$ 37000) components in a rare source in this family of outbursts, V899 Mon, using SALT-HRS.

\section{Overview of V899 Mon}

An outburst of V899 Mon (a.k.a. IRAS 06068-0641) located near the Monoceros R2 region (d 905 pc; [6]) was first discovered by the Catalina Real-Time Transient Survey (CRTS) and reported by [9]. They announced the source as a FUor candidate based on the constant brightening it has undergone since 2005. Our long-term multi-wavelength observations of this source started in 2009 and we could track the source undergoing transition from the first outburst phase to quiescent phase, and returning to a second outburst after spending a year in quiescence [7].

Figure 1 (i) shows the R band light curve evolution of V899 Mon. Our long-term ( $\sim 5$ years) medium resolution spectral monitoring of V899 Mon source shows outflows are overall correlated with the accretion rate in an outburst. But, in short timescales, there are significant fluctuations in the outflows. As shown in Figure 1 (ii), our first spectrum taken during the 1st outburst does not show any significant P-Cygni outflows. The outflows suddenly increased to its strongest level just before V899 Mon transitioned to quiescence. During quiescence the outflows completely disappeared. They were absent in the beginning of the 2 nd outburst, but its strength slowly increased as the 2 nd outburst progressed.

\section{SALT-HRS Observation and Reduction}

We acquired a high resolution $(\mathrm{R} \sim 37000)$ spectrum of V899 Mon during its second outburst phase on 2014 December 22 using the Southern African Large Telescope High Resolution Spec- 


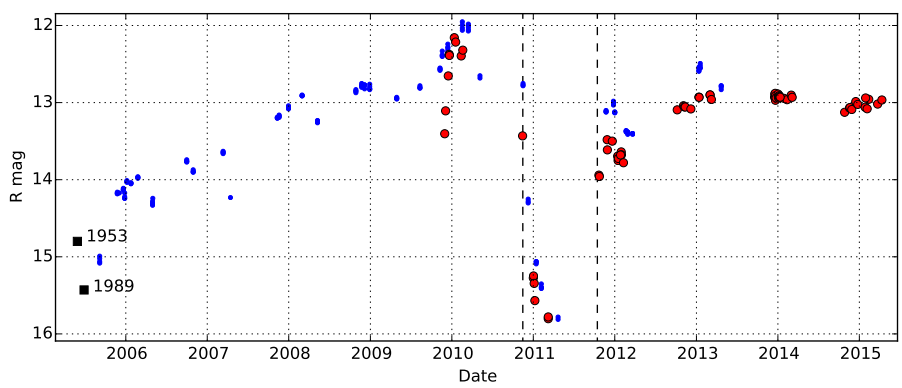

(i)

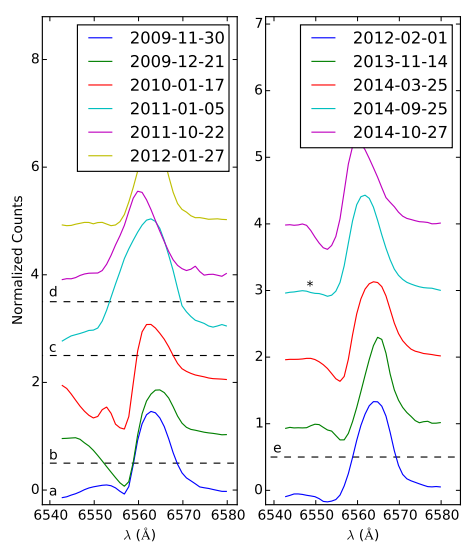

(ii)

Figure 1: (i) The light curve of V899 Mon showing its first outburst, short quiescent phase and current ongoing second outburst phase. Red circles are $R$-band magnitudes from our observations and blue circles are CRTS magnitudes converted to $R$ magnitudes. POSS-1 and POSS-2 epochs' $R$ magnitudes are also shown for comparison as black squares. (ii) Selected samples of $\mathrm{H} \alpha$ emission line profiles from different phases. a) First outburst phase, b) Heavy outflow during the end of first outburst phase, c) Quiescent phase, d) Transition phase , e) Second outburst phase.

trograph (SALT-HRS) [3]. Apart from target frames and flats, a ThAr arc lamp spectrum was also taken for wavelength calibration.

Apertures for each order were extracted by adaptive thresholding of the flats. Sky lines were aligned and subtracted from the extracted spectrum. All the data reduction was done by writing a general SALT-HRS reduction tool in Python, and it is available to the public ${ }^{1}$. Observed velocities were finally translated to heliocentric velocities by adding $+0.92 \mathrm{~km} / \mathrm{s}$ calculated using rvcorrect task in IRAF.

\section{Outflow structure near core}

The time evolution of the heavy outflows just before V899 Mon stopped its first outburst are consistent with a magneto-spheric accretion instability, which temporarily paused the accretion outburst into a quiescence. In such a scenario, probing the innermost region of outflowing gas is crucial to understand the dynamics of these winds/outflows. The blue-shifted absorption components in these classical P-Cygni profile of the $\mathrm{H} \alpha$ line, shown in Figure 2, are due to absorption by the outflowing gas from the innermost region. Hence, any structures in the outflow gas are directly reflected in the profile shape of the absorption component. The red-shifted part of the line profile peaks at $+18 \mathrm{~km} / \mathrm{s}$ and has smooth wings with an extra broad component reaching up to a velocity of $+420 \mathrm{~km} / \mathrm{s}$. The blue-shifted outflow absorption component extends up to $-722 \mathrm{~km} / \mathrm{s}$. We could see structures in absorption at $-648 \mathrm{~km} / \mathrm{s},-568 \mathrm{~km} / \mathrm{s},-460 \mathrm{~km} / \mathrm{s},-274 \mathrm{~km} / \mathrm{s},-153 \mathrm{~km} / \mathrm{s},-100 \mathrm{~km} / \mathrm{s}$ and $-26 \mathrm{~km} / \mathrm{s}$. Similar outflow velocity components are seen in the Ca II IR triplet lines also. These outflow absorption lines are optically thin and hence can be used to estimate the column density in the outflow to be $\mathrm{N}_{H} \sim 3.8 \times 10^{20} \mathrm{~cm}^{-2}$. These components are consistent with what we expect

\footnotetext{
${ }^{1}$ https://github.com/indiajoe/SALTHRS
} 
from a magnetic reconnection driven wind rather than a steady stellar wind. Our ongoing HRS multi epoch observation will help us in constraining the timescale and the velocity evolution of each of these sub-components.

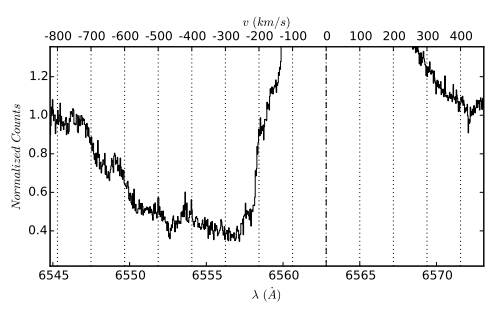

(a) $\mathrm{H} \alpha \lambda 6563$

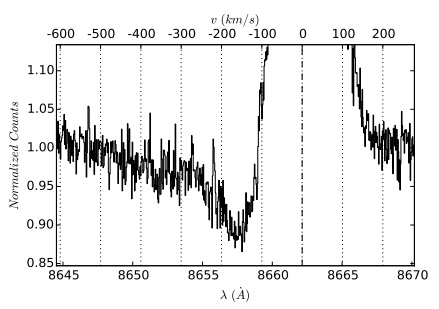

(b) Ca II $\lambda 8662$

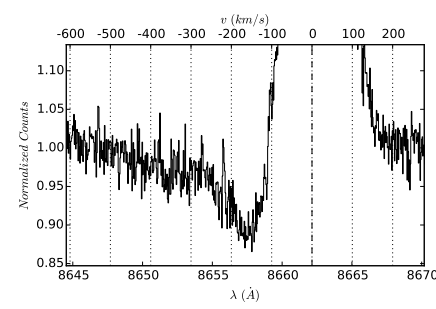

(c) Ca II $\lambda 8662$

Figure 2: $\mathrm{H} \alpha$ and Ca II IR triplet P-Cygni absorption line profiles of V899 Mon observed on 2014 December 22 using SALT-HRS.

\section{Outflow structure in large scale}

Using HRS we could also resolve the forbidden lines [OI] 6300, $6363 \AA$ and [Fe II] $7155 \AA$ originating in low density shocked regions of jets/outflows. These lines show a very interesting plateau profile (see Figure 3). [OI] $6300 \AA$ shows a strong red-shifted emission at $+25 \mathrm{~km} / \mathrm{s}$ with an FWHM of $21 \mathrm{~km} / \mathrm{s}$. The emission in the blue-shifted part extends up to $-450 \mathrm{~km} / \mathrm{s}$. Since the forbidden line emissions are optically thin, their fluxes are directly proportional to the column density of the emitting species. The plateau profile implies an almost equal column density in each projected velocity component in the outflow. From the line flux and ratios we could estimate the outflow rate to be $2 \times 10^{-7} \mathrm{M}_{\odot} / \mathrm{yr}$, temperature $\sim 9000 \mathrm{~K}$ and density $\mathrm{N}_{e} \sim 2 \times 10^{5}$ to $4 \times 10^{6} \mathrm{~cm}^{-3}$.

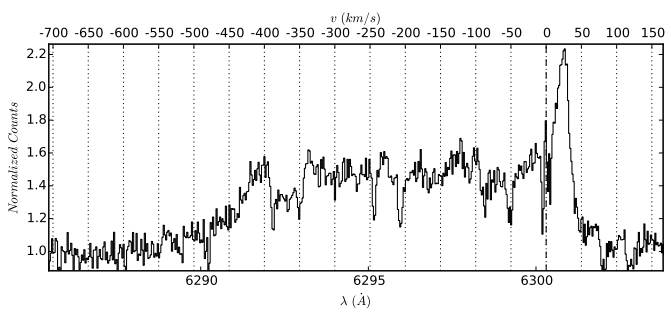

(a) $[\mathrm{OI}] \lambda 6300$

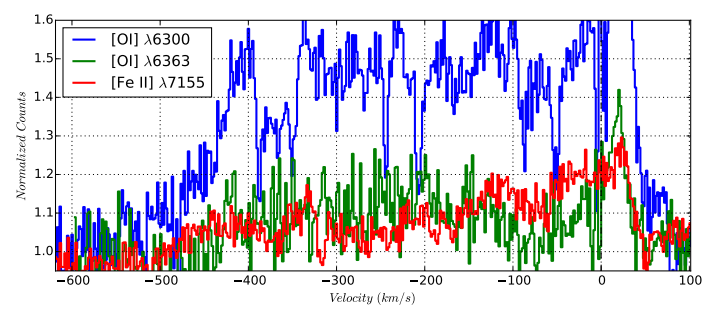

(b) $[\mathrm{OI}] \lambda 6363$ and [FeII] $\lambda 7155$

Figure 3: (a) Peculiar plateau structure in the forbidden line [OI] $\lambda 6300$. (b) $[\mathrm{OI}] \lambda 6363$ and $[\mathrm{FeII}] \lambda 7155$ emission line profile structures plotted underneath the [OI] $\lambda 6300$ profile of V899 Mon to show the consistency in the overall velocity structure.

\section{Conclusions}

Our long-term monitoring has suggested instabilities in the magneto-spheric accretion region to be the plausible cause of the break in the first outburst of V899 Mon. SALT-HRS spectrum taken with $\mathrm{R} \sim 37000$ could resolve multiple velocity components in the P-Cygni profile, which 
traces the outflow wind from the magneto-spheric accretion region (with velocities upto $-722 \mathrm{~km} / \mathrm{s}$ ). The HRS spectrum could also resolve forbidden line emissions originating in shocked regions by outflows/jets, revealing a very peculiar plateau structure, with velocity components extending up to $-500 \mathrm{~km} / \mathrm{s}$.

\section{Acknowledgments}

We would like to thank Dr. Brent Miszalski, and the entire SALT team for conducting SALT observations.

\section{References}

[1] P. Ábrahám, A. Juhász, C. P. Dullemond, et al. Episodic formation of cometary material in the outburst of a young Sun-like star, Nature, 459, 224, 2009

[2] I. Baraffe, E. Vorobyov, \& G. Chabrier, Observed Luminosity Spread in Young Clusters and FU Ori Stars: A Unified Picture, ApJ, 756, 118, 2012

[3] D. G. Bramall, R. Sharples, L. Tyas, et al., The SALT HRS spectrograph: final design, instrument capabilities, and operational modes, in Society of Photo-Optical Instrumentation Engineers (SPIE) Conference Series, Vol. 7735, 4, 2010

[4] N. J. Evans II, M. M. Dunham, J. K. Jorgensen, et al., The Spitzer c2d Legacy Results: Star-Formation Rates and Efficiencies; Evolution and Lifetimes, ApJ Supplement Series, 181, 321, 2009

[5] G. Ioannidis, \& D. Froebrich, YSO jets in the Galactic plane from UWISH2 âĂŞ II. Outflow luminosity and length distributions in Serpens and Aquila, MNRAS, 425, 1380, 2012

[6] M. Lombardi, J. Alves, \& C. J. Lada, 2MASS wide field extinction maps: IV. The Orion, Monoceros R2, Rosette, and Canis Major star forming regions, A\& A, 535, A16, 2011

[7] J. P. Ninan, D. K. Ojha, T. Baug, et al., V899 Mon: An Outbursting Protostar With Peculiar Light Curve And Its Transition Phases, ApJ, 815, 4, 2015

[8] A. Scholz, D. Froebrich, \& K. Wood, A systematic survey for eruptive young stellar objects using mid-infrared photometry, MNRAS, 430, 2910, 2013

[9] P. Wils, J. Greaves,A. J. Drake , \& M. Catelan, IRAS 06068-0641 and IRAS 06068-0643, Central Bureau Electronic Telegrams, 2033, 1, 2009 